\title{
Young Children's Inductive Generalizations about Similarities between Siblings or Classmates
}

\author{
Bradford H. Pillow ${ }^{1} \&$ RaeAnne M. Pearson ${ }^{1}$ \\ ${ }^{1}$ Department of Psychology, Northern Illinois University, DeKalb, Illinois, United States \\ Correspondence: Bradford H. Pillow, Department of Psychology, Northern Illinois University, DeKalb, IL \\ 60115, United States. E-mail: pillow@niu.edu
}

Received: October 4, 2016

Accepted: November 10, 2016

Online Published: December 4, 2016

doi:10.5539/jedp.v7n1p29

URL: http://dx.doi.org/10.5539/jedp.v7n1p29

\begin{abstract}
This study investigated 3- to 5-year-olds' inductive generalizations about similarities between siblings. Children were presented with contrasts in appearance and either sibling or classmate status and asked to generalize either biological properties or behaviors. Performance did not differ from chance for judgments about siblings, but children generalized on the basis of appearance more than classmate status. Thus, young children do not necessarily expect siblings to share biological and behavioral characteristics, but do regard sibling status as different from an arbitrary social relationship such as classmate status.
\end{abstract}

Keywords: biological knowledge, induction, kinship

\section{Introduction}

The concepts of family resemblance and inheritance are central to debate about the development of children's intuitive biological knowledge (Solomon \& Zaitchik, 2012). Carey and colleagues (Carey, 1985, 1988; Solomon, Johnson, Zaitchik, \& Carey, 1996) have argued that young children (a) do not recognize biology as a distinct and coherent conceptual domain, and (b) do not understand inheritance as the biological transmission of characteristics from parents to offspring. According to this view, young children recognize family resemblance (i.e., children expect offspring to resemble their parents), but do not understand resemblance as resulting from a biological causes, rather than social or environmental conditions. In contrast, others (e.g., Gelman \& Wellman, 1991; Springer, 1992; Springer \& Keil, 1989) have argued that preschool children do understand the biological inheritance of traits. Studies of children's understanding of resemblance and inheritance have examined reasoning about resemblance between parents and offspring. In the present study, we extended this work by examining young children's reasoning about similarities between siblings.

Previous studies have asked children about either the inheritance of species-general characteristics or the inheritance of individual differences within a species. For example, Gelman and Wellman (1991) told children ages 3- to 6-years of age about an animal that had been separated from its parents and other members of its species and raised with another species. Children were asked if the animal would have the characteristics of its own species or the characteristics of the species among which it was raised, e.g., would a cow raised among pigs moo or oink? By 4-years of age children attributed to the animal the characteristics of the animal's own native species more than the characteristics of the species with which the animal had been raised. Springer (1992) investigated children's beliefs about individual differences within a species. Springer (1992) presented children ages 4- to 8-years with a picture of an animal and described it as having a particular characteristic, e.g., a horse that had hair inside its ears. Then children were shown a picture of second animal that looked similar to the first animal, but was described as an unrelated friend, and a picture of third animal that looked different from the first animal, but was described as its baby. When asked if the second and third animals shared the first animal's characteristic, children attributed the characteristic to the baby more frequently than to the friend. However, Solomon et al. (1996) argued that such evidence indicates that young children understand that offspring resemble their parents, but does not necessarily demonstrate that children understand biological inheritance.

According to Solomon et al. (1996), having a concept of inheritance requires that children recognize (a) that offspring resemble their parents, and (b) that family resemblance results from biological causation. A biological concept of inheritance would include recognition that inherited traits are related to birth rather than to social 
experience. Therefore, Solomon et al. (1996) assessed children's understanding of inheritance by presenting them with an adoption scenario in which a king/queen's child was adopted by a shepherd/shepherdess (or vice versa). Children were asked if the child shared the physical traits and beliefs of the biological parent or the adoptive parent. Solomon et al. (1996) reasoned that if children understand inheritance as a biological process related to birth, children should attribute the biological parent's physical traits to the child, but attribute the adoptive parent's beliefs to the child. However, young children did not recognize that an adopted child is likely to resemble a biological parent for physical traits, such as eye color, and likely to resemble an adoptive parent for learned characteristics, such as beliefs about skunk's ability to see in the dark. Because children did not consistently differentiate physical traits and beliefs in their judgments until 7-years of age, Solomon et al. (1996) concluded that younger children do not recognize family resemblance as inherited through a biological process that is distinct from teaching and learning. Solomon (2002) reported that 4- and 5-year-olds judged an adoptive child as equally likely to share a biological parent's shirt color and race. Nevertheless, Solomon and Johnson (2000) found that following a twenty-minute training session that emphasized the role of genes in inheritance, many 5- and 6-year-olds improved in performance on an adoption task, though the majority of children still did not differentiate between physical traits and beliefs.

In the present study we investigated young children's reasoning about family resemblance between siblings. Understanding resemblance between siblings presents children with a different challenge, compared to understanding resemblance between parents and offspring. Because siblings often are similar in age, the physical resemblance between siblings, especially during childhood, might be more readily evident to children than is the resemblance between a parent and a young child. However, the nature of the kinship relation between siblings might be less apparent than the relationship between parent and child. Whereas parents and children are related directly through biological inheritance, siblings are related indirectly, via shared parentage. Thus, the notion that siblings are biologically related, as well as socially related, might be less apparent to children. We asked 3-, 4-, and 5-year-olds to reason about biological and behavioral characteristics of siblings and non-siblings.

Our procedure was based on a triad task that Gelman and Markman (1986) used to study young children's patterns of inductive generalization. Gelman and Markman (1986) presented 4-year-olds with sets of three pictures. Each triad included two test items and a target. The target belonged to the same category as one test item differed in appearance, and the resembled the other test item, but belonged to a different category (e.g., the test items were a flamingo (labeled as a bird) standing on one foot and a bat with wings outstretched, and the target was a blackbird with wings outstretched). Children were taught an unfamiliar property for each test item (e.g., the bird (flamingo) feeds its babies mashed up food; the bat feeds its babies milk). Then children were asked which property was characteristic of the target. Children based the majority of their inferences on category membership (e.g., responding that the blackbird behaves like the flamingo rather than the bat). Likewise, subsequent research indicates that when given a choice of basing inductive generalizations on shared category membership or similarity in perceptual appearance, young children typically generalize on the basis of category membership (Gelman, 2003; Gelman \& Davidson, 2013). In the present study, we examined children's use of sibling status as a basis for inductive generalizations. In the Sibling condition, we presented children with triads with pictures of three children. The first two pictures portrayed children were unrelated and differed in appearance. Participants taught about either a biological or behavioral property of these two children (e.g., "This boy has fibros in his blood. This boy has neutros in his blood."). The participants were shown a picture of third child who looked similar to one of the first two children, but was the sibling of the other child. Participants were asked if the third child shared the same property as his or her dissimilar sibling or shared the property of the unrelated child who he or she resembled in outward appearance. As a comparison, in the Classmate condition, the third child in each triad was a classmate of one of the first two children, but did not resemble the classmate, and resembled the other child, but was not a classmate of that child. Because being classmates is a temporary, and more or less serendipitous, relationship, classmate status should not support inductive inferences to the same degree as kinship.

The design of the study allowed us to distinguish among patterns of attribution based on outward appearance, biological kinship, or social relations. If young children generalize unseen biological or behavioral characteristics on the basis of similarity in outward appearance, then in both the Sibling and Classmate conditions, participants should judge that the third child in each triad shares the property of the child who similar in appearance, but unrelated. If children regard family resemblance as biologically-based and also recognize siblings as biologically related, then in the Sibling condition participants should generalize from sibling to sibling within each triad, but should generalize on the basis of similarity in appearance in the Classmate 
condition. If children lack a biological understanding of kinship and regard being siblings or classmates as equivalent social relationships, they should generalize on the basis of sibling or classmate status equally often and more often than they generalize on the basis of appearance.

\section{Method}

\subsection{Participants}

Thirty two 3 year olds (mean age 3 years, 8 months; age range 3 years, one month to 3 years, 11 months; 17 girls and 15 boys), 324 year olds (mean age 4 years, 5 months; age range 4 years to 4 years, 10 months; 16 girls and 16 boys), and 325 year olds (mean age 5 years, 5 months; age range 5 years, 1 month to 5 years, 9 month; 18 girls and 14 boys) participated. The children were students at preschools in either suburban or rural towns in the northern Illinois region of the United States. Children in the Classmate condition participated in a related study reported by Pillow, Pearson, and Allen (2015). In addition, 10 adults (mean age 21 years; range 19-23 years; 7 women and 3 men) participated in a manipulation check prior to the main experiment. The adults were students enrolled in an upper level undergraduate psychology course at a public university.

\subsection{Materials}

Eight sets of computer generated pictures of children were used for the main test procedure. Each set was a triad, with pictures of two children (the test pictures) on the same page placed above a picture of a third child on a separate page (the target pictures). On the top page, the two test children stood side by side. The two children differed in hair color, hair length, eye color, facial features, and shirt color, but had identical bodies, posture, pants, and shoes. On the second page, the target child had the same hair color, eye color, and facial features as one of the two test children, but had hair of an intermediate length and a different color shirt than either of the test children. This target child matched the two test children in body, posture, pants, and shoes. Across the eight sets of pictures, hair color, hair style, eye color, facial features, and shirt color were varied so that the individuals in each triad were distinct from the individuals in the other triads. Because race and ethnicity of the depicted children were not variables under investigation, these variables were held constant by making all children in the pictures Caucasian in appearance. Additional sets of pictures were used in the familiarization procedure.

\subsection{Procedure}

Each child participated in one of two conditions: Sibling or Classmate. In each condition, children began with a brief familiarization procedure. The familiarization procedure introduced children to information about either sibling or classmate status, and similarities and differences in appearance. This procedure also accustomed children to pointing to pictures. In the Sibling condition, there were two familiarization trials. For the first trial, half of the children were shown two pairs of pictures of boys, and the other half were shown two pairs of pictures of girls. One of pair of pictures differed in appearance but were labeled as siblings (e.g., "Here are two boys. This boy and this boy are brothers."). The pictures in the other pair were similar in appearance, but were labeled as unacquainted with each other (e.g., "Here are two boys. This boy and this boy don't know each other."). Children were asked, "Can you point to the boys that are brothers?" and "Can you point to the boys that don't know each other?" For the second familiarization trial, children were shown the two pairs of pictures from the first two trials. The experimenter pointed to the first pair and said, "I think these two look kind of the same", and then pointed to the second pair and said "I think these two look different". Then the experimenter asked, "What do you think? Can you point to the ones that look kind of the same? And can you point to the ones that look different?". In the Classmate condition, there were two familiarization trials. The procedure in the Classmate condition was the same as in the Sibling condition, except that for the first trial one pair of pictures was labeled as classmates rather than siblings.

Following the familiarization, there were eight test trials. On each trial children were shown a set of pictures, taught either a biological or behavioral characteristic for each of the two test children (test child A and test child B) and then asked if the target child had the same characteristic as test child A or test child B. In Sibling condition all three children (target and test) were described as being the same gender. The target child was described as being a brother or sister of either test child A or test child B, but visually resembled the test child that he or she was not related to. For example, children were told, "This girl has telos in her body. This girl has brevies in her body. Now here's someone else. She looks kind of like this girl. And she's this girl's sister". Likewise, in the Classmate condition, the target child resembled one of the test children, but was a classmate of the other, dissimilar test child. For example, children were told, "This boy likes to share a lot. This boy likes to laugh a lot. Now here's someone else. He looks kind of like this boy here. And he's in this boy's class at school”. 
All of the behaviors mentioned on behavioral trials were positive and socially desirable. The items were based on those used by Gelman, Collman, and Maccoby (1986) and Pillow et al. (2015). Biological and behavioral items are shown in the appendix. For all trials, both the target child's similarity in appearance with one of the test children, and the target child's sibling or classmate status with other test child, were explicitly mentioned.

In each condition, there were four biological trials and four behavioral trials. Biological and behavioral trials were presented in alternating blocks of two trials of each type, with half of the children in each condition beginning with two biological trials, and half beginning with two behavioral trial. The order of mention of the sibling and non-sibling, or classmate and non-classmate, was alternated across trials. For half of the trials in the Sibling and Classmate conditions, all three children were boys, and for the other half the children were girls.

A manipulation check was included to ensure that the pictures intended to be similar in appearance were perceived as more similar than were the pictures intended to be different in appearance. For the manipulation check, adults were shown pairs of pictures and asked to rate their similarity. The eight triads from the main procedures were used. In each triad, there are three possible pairings of pictures. Adults were shown each of the three pairings for each triad, resulting in 24 pairs of pictures. In addition, 12 pictures were paired with an identical copy of themselves to create matching pairs. Altogether there were 36 pairs of pictures, which were presented in random order. First, adults were told, "You will be seeing pictures of people, presented two at a time. For each pair of pictures, please rate how similar the two people are to each other". Then they were shown a five point rating scale, with one labeled as "different", 3 labeled as "somewhat similar", and 5 labeled as "highly similar". Next participants were told, "Use this five point scale to rate the similarity of the two people in each pair of pictures". Participants viewed and rated the pairs of pictures at their own pace.

\section{Results}

For the manipulation check, adults rated Matching pictures $(M=5.00)$ as significantly more similar than the Similar pictures $(M=3.52), t(9)=10.15, p<.001$, and rated the Similar pictures as significantly more similar than the Dissimilar pictures $(M=1.95), t(9)=8.44, p<.001$. Thus, adults perceived the Similar pictures as being more alike visually than the Dissimilar pictures.

Each child was a given a score from 0-4 for biological trials and a score from 0-4 for behavioral trials. Scores corresponded to the number of trials on which the child responded that the target would have the same trait as the test child who was a sibling (Sibling condition), or was a classmate (Classmate condition). Results are shown in Table 1. The data were analyzed in two steps. First, to examine possible effects of age, condition (Sibling versus Classmate), and the type of trait children were asked about (Biological versus Behavioral) a $3 \times 2 \times 2$ (Age $\mathrm{x}$ Condition $\mathrm{x}$ Trait) ANOVA with Trait as a repeated measures factor was conducted. Second, children's scores were compared to chance expectancies. The ANOVA yielded a significant effect of Condition, $F(1$, 90) $=4.13, p<.05$, partial $\eta^{2}=0.044, M S E=1.92$, with higher scores in the Sibling Condition than in the Classmate condition. For each condition, performance was compared to chance responding ( 2 out of 4 trials) for both biological and behavioral traits. Because the Age x Condition x Trait ANOVA did not yield any significant effects of Age, the three age groups were combined for these comparisons. In the Sibling condition, performance did not differ from chance for either biological, $t(47)=0.69, p>.05$ or behavioral traits, $t(47)=0.91, p>.05$. In the Classmate condition, children attributed both biological, $t(47)=3.93, p<.001$, and behavioral traits, $t(47)=2.45$, $p<.05$, on the basis of shared classmate status significantly less often than would be expected by chance.

Table 1. Mean number of category match responses by age, condition, and property

\begin{tabular}{|c|c|c|c|c|c|c|c|c|}
\hline \multirow[b]{3}{*}{ Age } & \multicolumn{4}{|c|}{ Sibling } & \multicolumn{4}{|c|}{ Classmate } \\
\hline & \multicolumn{2}{|c|}{ Biology } & \multicolumn{2}{|c|}{ Behavior } & \multicolumn{2}{|c|}{ Biology } & \multicolumn{2}{|c|}{ Behavior } \\
\hline & Mean & $\mathrm{SD}$ & Mean & SD & Mean & SD & Mean & SD \\
\hline 3-years & 1.75 & 1.18 & 1.63 & 1.31 & 1.75 & 1.00 & 2.06 & 1.12 \\
\hline 4-years & 2.25 & 1.25 & 2.06 & 0.93 & 1.00 & 1.15 & 1.44 & 1.21 \\
\hline 5-years & 1.63 & 1.31 & 1.88 & 1.09 & 1.31 & 1.20 & 1.19 & 1.28 \\
\hline
\end{tabular}

Note. Scores range from $0-4$. 


\section{Discussion}

We examined 3- to 5-year-old children's use of information about sibling status, classmate status, and similarity in physical appearance when making inductive generalizations about biological and behavioral characteristics. Although children in the Sibling condition generalized characteristics from one sibling to another more often than children in classmate condition from one classmate to another, children's performance in the Sibling condition did not differ from chance for either biological or behavioral characteristics. Thus, in the Sibling condition children did not treat kinship as more important than similarity in appearance when making inductive generalizations. Therefore, in the Sibling condition children did not demonstrate a biologically-based understanding of family resemblance. In the Classmate condition, most of the time children generalized both biological behavioral characteristics on the basis of similarity in appearance rather than on the basis of classmate status. Across the two conditions children did not demonstrate consistent use of either appearance or kinship as the sole basis for induction, nor did children generalize equally on the basis of sibling and classmate relationships. Instead, children seemed to consider both appearance and social relationships, weighting a sibling relationship as roughly equal in inductive strength to similarity in appearance, but regarding a classmate relationship as a much weaker basis for induction than similarity in appearance.

The present results are consistent with Solomon et al.'s (1996) conclusion that young children do not understand family resemblance as resulting a biological process of inheritance that is related to birth. Solomon et al. (1996) found that prior to 7-years of age children did not consistently attribute a biological parent's physical traits, but an adoptive parent's beliefs, to a child who had been adopted at birth. In the present study, we examined children's expectations concerning family resemblance between siblings, but we did not investigate children's beliefs about biological inheritance. We found that 3- to 5-year-old children did not expect siblings to share either unseen internal biological properties or behavioral characteristics. Thus, in contrast to previous findings that young children expect offspring to resemble their parents, we did not find evidence that young children expect siblings to resemble each other. However, in studies of parent-offspring family resemblance, the biological traits presented have included a mix of observable external features and hidden internal features. Young children often may base generalizations on visual or global similarity rather than more abstract relationships (Deng \& Sloutsky, 2012; Jones \& Smith, 1993; Sloutsky \& Fisher, 2004). Thus, the notion that family members resemble each other in outward appearance may be more familiar and accessible to young children compared to the idea that family members might possess similar internal features. Children in our study may have failed to evidence a belief in family resemblance either because (a) children recognize family resemblance between parents and offspring before they recognize resemblance between siblings, or (b) children recognize resemblance in external features before they expect resemblance in internal features.

Young children also might be more aware of individual differences in external features than internal features. Research on category-based induction indicates that children expect members of the same species or general biological category to share both internal features and typical behavioral characteristics (Gelman, 2003; Gelman \& Davidson, 2013). Furthermore, when asked about cases of cross-species adoption, children ages 4 years and older expected an adopted animal to share the characteristics typical of its species more than the characteristics of the species with which it had been raised (Gelman \& Wellman, 1991). Learning about individual differences within a species may present children with a different challenge than reasoning about the typical shared characteristics of a species. Individual differences in external features should be more accessible to both perception and thought than differences in internal features, and to the extent that children expect members of species to share non-obvious features, the possibility of individual differences in such features may not come to mind readily. Therefore, the use of internal biological features in the present study may have contributed to children's failure to base inductions on kinship more than on similarity in appearance.

The present study was an initial effort to extend research on children's beliefs about family resemblance to include reasoning about siblings. The results indicate that young children do not necessarily expect siblings to share biological and behavioral characteristics, but do regard sibling status as different from an arbitrary social relationship such as classmate status. These results suggest directions for additional research. Future studies could (a) directly compare children's reasoning about sibling relationships with reasoning about parent-offspring relationships, (b) examine children's reasoning about sibling adoption scenarios to assess beliefs about inheritance, (c) compare children's reasoning about siblings with reasoning about other close social relationships, such as friends or neighbors, (d) examine children's generalization of a variety of characteristics, including external and internal characteristics, behaviors, preferences, and beliefs, and (e) trace developmental progress in 
understanding family resemblance and inheritance by comparing young children's reasoning about siblings with older children's reasoning.

\section{Acknowledgments}

We thank the children, parents, and schools who participated in this study.

\section{References}

Carey, S. (1985). Conceptual change in childhood. Cambridge, MA: MIT Press.

Carey, S. (1988). Conceptual differences between children and adults. Mind \& Language, 3, 167-181. http://dx.doi.org/10.1111/j.1468-0017.1988.tb00141.x

Deng, W., \& Sloutsky, V. M. (2013). The role of linguistic labels in inductive generalization. Journal of Experimental Child Psychology, 114, 432-455. http://dx.doi.org/10.1016/j.jecp.2012.10.011

Gelman, S. A., \& Davidson, N. S. (2013). Conceptual influences on category-based induction. Cognitive Psychology, 66, 327-353. http://dx.doi.org/10.1016/j.cogpsych.2013.02.001

Gelman, S. A. (2003). The essential child. New York: Oxford University Press. http://dx.doi.org/10.1093/acprof:oso/9780195154061.001.0001

Gelman, S. A., \& Markman, E. M. (1986). Categories and induction in young children. Cognition, 23, 183-209. http://dx.doi.org/10.1016/0010-0277(86)90034-X

Gelman, S. A., Collman, P., \& Maccoby, E. E. (1986). Inferring categories from properties vs. inferring properties from categories: The case of gender. Child Development, 57, 396-404. http://dx.doi.org/10.2307/1130595

Gelman, S. A., \& Wellman, H. M. (1991). Insides and essences: Early understandings of the nonobvious. Cognition, 38, 213-244. http://dx.doi.org/10.1016/0010-0277(91)90007-Q

Jones, S. S., \& Smith, L. B. (1993). The place of perception in children's concepts. Cognitive Development, 8 , 113-139. https://dx.doi.org/10.1016/0885-2014(93)90008-S

Pillow, B. H., Pearson, R. M., \& Allen, C. (2015). Young children's inductive generalizations about social categories: When is gender essential? Merrill-Palmer Quarterly, 61, 441-467. http://dx.doi.org/10.13110/merrpalmquar1982.61.4.0441

Sloutsky, V. M., \& Fisher, A. V. (2004). Induction and categorization in young children: A similarity-based model. Journal of Experimental Psychology: General, 133, 166-188. https://dx.doi.org/10.1037/0096-3445.133.2.166

Solomon, G. E. A. (2002). Birth, kind and naive biology. Developmental Science, 5, 213-218. http://dx.doi.org/10.1111/1467-7687.00223

Solomon, G. E. A., \& Zaitchik, D. (2012). Folk biology. WIRES Cognitive Science, 3, 105-115. http://dx.doi.org/10.1002/wcs. 150

Solomon, G. E. A., Johnson, S. C., Zaitchik, D., \& Carey, S. (1996). Like father, like son: Young children's understanding of how and why offspring resemble their parents. Child Development, 67, 151-171. http://dx.doi.org/10.1111/j.1467-8624.1996.tb01726.x

Solomon, G. E. A., \& Johnson, S. C. (2000). Conceptual change in the classroom: Teaching young children to understand biological inheritance. British Journal of Developmental Psychology, 18, 81-96. http://dx.doi.org/10.1348/026151000165580

Springer, K. (1992). Children's awareness of the biological implications of kinship. Child Development, 63, 950-959. http://dx.doi.org/10.1111/j.1467-8624.1992.tb01674.x

Springer, K., \& Keil, F. C. (1989). On the development of biologically specific beliefs: The case of inheritance. Child Development, 60, 637-648. http://dx.doi.org/10.2307/1130729 


\section{Appendix}

Biological items:

Has fibro/neutros in his/her blood.

Has telos/brevies in his/her body.

Has a sarto/lattis in his/her body.

Has transfa/protho in his/her blood.

Behavioral items:

Always works hard on puzzles/Always cleans up toys at school.

Likes to share a lot/laugh a lot.

Always says please and thank you/Always holds puppies and kittens carefully.

Likes to follow rules/Likes to meet new people.

\section{Copyrights}

Copyright for this article is retained by the author(s), with first publication rights granted to the journal.

This is an open-access article distributed under the terms and conditions of the Creative Commons Attribution license (http://creativecommons.org/licenses/by/4.0/). 